\title{
EDITORIAL
}

\section{REDES DE INVESTIGACIÓN}

no de los términos que está en boga en el ambiente académico es el de redes de investigación, cuya definición se puede tornar compleja, pero que en síntesis lo que pretende es la interrelación entre los académicos, la empresa y el Estado, que congregados por intereses comunes y con la disposición de realizar un trabajo colaborativo, en el que se conjugue un aporte de conocimientos, habilidades y capacidades que impulsen sinérgicamente soluciones a problemas y temas estratégicos que contribuyan con el desarrollo del país.

Esta relación interdisciplinaria, mediada por el conocimiento y prestigio reconocido sobre las temáticas abordadas facilita la colaboración en la gestión de la investigación, en cuanto a la planeación, diseño, realización y seguimiento de los proyectos, lo que permite el crecimiento y consolidación de la Red.

No obstante esta interesante y promisoria intencionalidad de las redes de investigación, al revisar la Política de Ciencia y Tecnología con respecto al tratamiento que se le da a éstas, se observa que no hay referencias explícitas, aunque presenta un diagnóstico general referido a aspectos tales como:

- Bajos niveles de innovación de las empresas.

- Débil institucionalidad en el Sistema Nacional de Ciencia, Tecnología e Innovación.

- Insuficiente recurso humano para la investigación y la innovación.

- Baja apropiación de la ciencia, la tecnología y la innovación.

- Ausencia de focalización en áreas estratégicas de largo plazo.

- Disparidades regionales en capacidades científicas y tecnológicas.

De allí surge el interrogante: ¿son estas también las razones que impiden la construcción de redes de investigación en Colombia?, o por el contrario, ¿la inexistencia o debilidad de las redes de investigación son las que generan la situación que se describe en el diagnóstico anterior? 
Al respecto, en el Documento CONPES número 3582 de abril de 2009 un grupo de ministerios y otras agencias del Estado le solicitaron al Ministerio de Educación Nacional, a COLCIENCIAS y al hoy Ministerio de las Tecnologías de la Información y las Comunicaciones, consolidar en el plazo de un año la red de instituciones académicas y de investigación RENATA, alcanzando un total de 15 centros de investigación conectados y fomentando el desarrollo de proyectos colaborativos en áreas estratégicas.

Hoy en día RENATA está integrada por tres miembros de Gobierno (Ministerio de Educación, Ministerio de Tecnologías de la Información y las Comunicaciones y Departamento Administrativo de Ciencia, Tecnología e Innovación, Colciencias) y las ocho redes académicas regionales (RADAR, RIESCAR, RUANA, RUAV, RUMBO, RUP, RUTA Caribe y UNIRED) a las cuales están conectadas más de 150 instituciones del país entre instituciones de educación superior, salud, cultura y centros de I+D+i.

Pero esto no parece ser suficiente para responder los interrogantes planteados, al punto que esta Red se ha convertido en un medio para apoyar el trabajo de los investigadores, sin que aún alcance mayor penetración entre sus grupos de interés, pese a que su creación surgió con el fin de "conectar, comunicar y propiciar la colaboración entre las instituciones académicas y científicas de Colombia con las redes académicas internacionales y los centros de investigación más desarrollados del mundo; y además; interconecta e incluye a todas las instituciones académicas y científicas del país, para fortalecer el desarrollo de la ciencia, la educación, la innovación, la cultura y la apropiación de las TIC". En este punto de la reflexión es donde se debe hacer un alto en el camino para pensar cuál es la carencia existente que impide que RENATA cumpla con unos objetivos que visibilicen esta interacción colaborativa de los miembros de una red de investigación.

Y retomando los interrogantes planteados, considerar la necesidad de elaborar y difundir una fundamentación y discurso alrededor de las Redes de Investigación en Colombia, comenzando, quizá, en el interior de las Instituciones de Educación Superior, de tal manera que se superen los individualismos y la desconexión entre las diferentes disciplinas y organizaciones, a fin de abordar los objetos de investigación científica, tecnológica y social de manera multidimensional.

Sólo así se podrán enfrentar los nuevos paradigmas y retos que se plantean en investigación, desarrollo e innovación y que nos sitúan verdaderamente frente a la complejidad. 